\title{
Performance Analysis for Data Service in Third Generation Mobile Telecommunication Networks
}

\author{
Aloizio P. Silva, Geraldo R. Mateus \\ Federal University of Minas Gerais \\ Department of Computer Science \\ Av. Antônio Carlos, 66-31270-010 - Pampulha - BH - MG - Brazil \\ \{aloizio, mateus\}@dcc.ufmg.br
}

\begin{abstract}
The data traffic in wireless networks for the third generation ( $3 G)$ mobile telecommunication systems should take into account a variety of services (voice, data, video) and environments (e.g.: private, outdoors, indoors) as well as the user mobility behavior. A good evaluation of measures of performance can help a system designer to make its strategic decisions concerning cell size and the number of channel frequencies allocated to each cell. In this paper we present an analysis of data services in a third generation mobile telecommunication networks based on simulation. In addition, we illustrate the need for a simulation in order to characterize the mix of several traffic types for capacity and quality of service (QoS) planning. We use the distributions heavy tailed Weibull and Pareto to simulate respectively, the data traffic and the resource occupation time for data service. Finally, we also comment some simulation results of third generation services where we analyze the QoS parameters of a mobile network, such as channel occupation time, handoff, new call blocking probabilities and traffic in Erlangs.
\end{abstract}

Keywords - Mobile Networks, Data Services, Third Generation, Quality of Service

\section{Introduction}

One of the driving forces of the next generation of wireless communication and computing networks is the promise of high-speed multimedia services. The next generation wireless mobile networks, known as third generation $(3 \mathrm{G})$ mobile telecommunication systems, should take into account a variety of services (voice, data, video) and environments (e.g.: private, outdoors, indoors) as well as the user mobility behavior. The great difference between the next and the current networks is their ability to provide higher-rate data service, which are expected to fuel a mobile device of new application. Third generation systems, such as Future Public Land Mobile Telecommunication System (FPLMTS or IMT-2000) and the Universal Mobile Telecommunication System (UMTS), promise to provide multimedia services to mobile and fixed users via wireless access to the global telecommunication infrastructure [25, 27, 32]. The UMTS idea is to enable Mobile Users (MUs) to access differentiated services of 'anywhere', and 'anytime', using a single telecommunication device.

The evolution of cellular mobile networks, pushed by a rapidly increasing number of subscribers, began in the field of telephony. Nevertheless, the evolution of mobile networks also followed a second path: the packet radio networks for mobile data communication. These networks provide packet oriented data transfer to and from a mobile station. In addition to the integration of speech and data services, the ongoing network evolution progress is directed towards personal communications and mobility. Building integrated services mobile networks can be realized either by improving the service profile offered by existing cellular systems, or by adding air interfaces to existing wired networks.

Traffic modeling in wireless telecommunication 
system has to deal with two main issues, the Radio Resource Management (RRM) scheme and the effect of the user mobility on the traffic volume per cell $[14,22,34]$. Data traffic in wireless telecommunication networks is emerging like one of aspects more important in third generation networks planning $[13,16,33,35]$. Thus a mix of several traffic types of different services has required more resources and QoS planning. Therefore, from the telecommunications point of view we can model the different traffic types like voice and data based on their behaviour.

Wireless data applications produce data that may have different characteristics from those of wired data applications and wireless voice data [18]. This article describes the simulation results of data service in mobile (cellular) telecommnunication networks, using the simulator named 'Simula2' [29, 30, 31], which take into account the self-similarity $[5,21,26]$ of the network traffic. We use the distributions heavy tailed Weibull and Pareto [17] to simulate the data traffic and the resource occupation time for data service, respectively. The simulation provides simple analytical results regarding the traffic of different services and also provides means to estimate the following parameters:

- telecommunication traffic volume,

- telecommunication traffic intensity,

- handoff blocking probability,

- call blocking probability, and

- channel occupation time.

When a MU wants to communicate with another MU, it must first obtain a channel from one of the base stations that hears depending on signal power. If a channel is available, it is assigned to the user. In the case that all the channels are busy, the new call is blocked. This kind of blocking is called new call blocking and it refers to blocking of new calls. On the other hand, the procedure of moving from one cell to another, while a call is in progress, is called handoff. While performing handoff, the MU requires that the base station of the destination cell will allocate it a channel. If no channel is available in the new cell, the handoff call is blocked. This kind of blocking is called handoff blocking and it refers to blocking of ongoing calls due to the mobility of the users [33]. Basically, the new call and handoff blocking probabilities is that allow to determine the Quality of Service (QoS) in cellular networks.

In addition, the traffic volume and intensity, and channel occupation time are also parameters that will allow to evaluate the system behavior and the resource utility. The time interval that a call is keeping busy a channel or a set of them is called channel occupation time. Then the traffic volume may be defined as the sum of the channel occupation time. The traffic intensity is a measure of the average occupancy of a channel during a specified period of time, normally a busy hour, measured in traffic units (Erlangs ${ }^{1}$ ). This is a dimensionless quantity and may be used to measured the time utilization of single or multiple channels. The results are obtained by assuming that voice and data service are available, while user moves.

The paper is organized as follows. In Section 2, the simulation organization and simulator characteristics are described. Section 3 reports the data traffic modeling used in the simulator. Simulation results and implications on QoS planning are given in Section 4. Finally, the conclusions and future directions, towards to the third generation systems are presented in Section 5.

\section{Simulation Organization}

We used the Manhattan Model $[4,23]$ that perfectly serves ours needs, and because of its simplicity, allows us to track hundreds of thousands of MUs. The square grid has $12.8 \mathrm{Km}$ sides. The streets are spaced every 128 meters, and are numbered $0,1,2, \ldots \mathrm{N}$, with each one accepting traffic in both directions. Speed of the MUs is controlled independently for the inner and the outer streets, typical values being 30 and $50 \mathrm{Km} / \mathrm{h}$ respectively. All cells have the same area, are circular and spread having minimum overlapping areas. The program allows us to define several areas within the city, which we call regions of interest. For implementation purposes we have limited these areas in:

- Traffic

- Residential

\footnotetext{
${ }^{1}$ Erlang or Erl corresponds to measured unit that determine the occupation rate of a channel, for example: to use a channel of 1 Erl means that the channel is been used in its maximum capacity all the time.
} 
- Business

- Shopping

- Park/Lake

The information entered can then be stored, allowing us to have several different templates for simulation purposes. Hence it is possible in the simulator to create as many MU categories as needed, and divide the whole population among the categories such as to reflect a real situation.

When the MUs are created within a simulation, the program randomly assigns one Home location and one Work location for each MU. We can also built timetable to schedule the MU's activities, which are used for traffic simulation. These timetables are defined using a simple ASCII editor. A typical example is shown on Figure 1.

In the Figure 1, we have 4 categories of MUs. The originating and the receiving call distributions can be defined by the words CALLDIST and RECDIST respectively. For each category we can also define different services and its connection average. These services can be voice, data or video. Each service has different requiring of bandwidth. To determine the bandwidth that will be used by each service, a different channel amount for each one has been allocated (i.e., two channel to WWW service and four channel to video service). To determine the traffic volume over the area under study, we define that:

- On voice service the call arrival process is assumed to be Poissonian while the call duration is exponentially distributed.

- On data service the call arrival process is assumed to be Pareto while the call duration follow the heavy tailed Weibull distribution.

- On video service the call arrival process is assumed to be Pareto while the call duration is exponentially distributed.

The simulator can also write a ASCII text file in disc. Figure 2 shows a example file.

We define in our simulator five types of events that can be processed by event manager as shown in Table 1 .

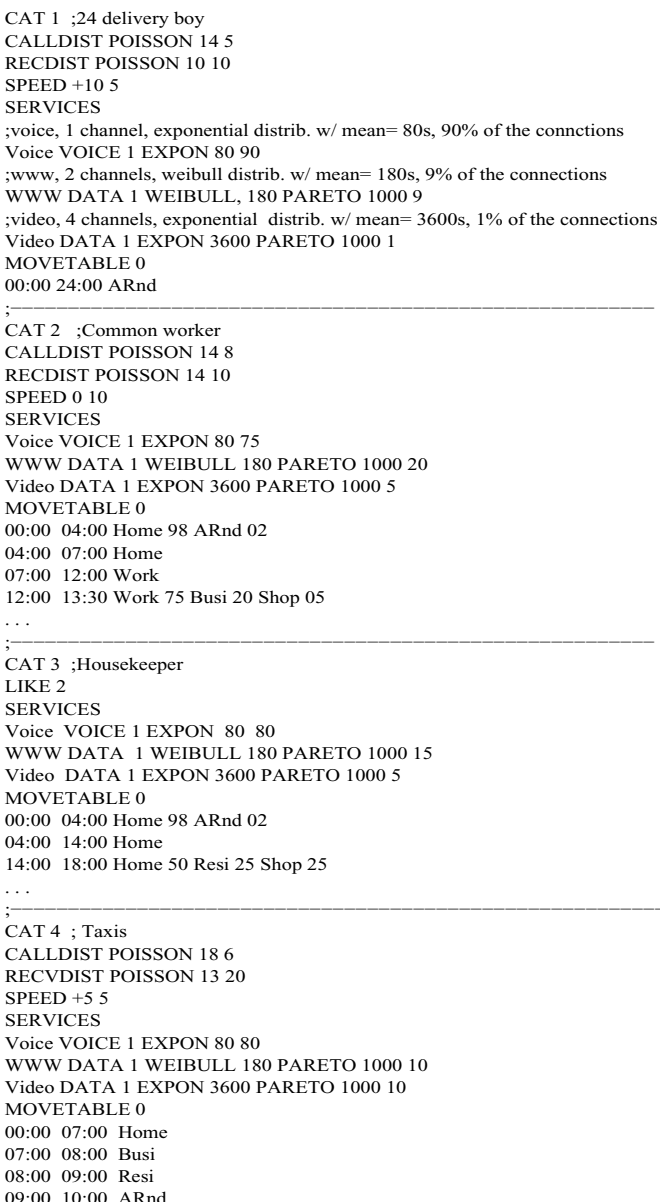

Figure 1. Timetable with location probability distribution given for 4 different MU categories

To handle the handoff request we used the Priority II model, defined in [15], that considers handoff queue. In $[20,21]$ and in numerous other studies $[5,7,8]$ network packet traffic appears "similar" when measured over a wide range of time scales. That is, the network traffic looks the same when measured over time intervals ranging [10]. Data traffic of this type is said to be self-similar or fractal in nature $[20,21]$. Selfsimilar traffic is very different from both conventional telephone traffic and from the currently accepted norm for model of packet traffic.

An important parameter of a self-similar process is the Hurst parameter $\mathrm{H}$. It is a parameter used to describe the degree of self-similarity and it can be esti- 


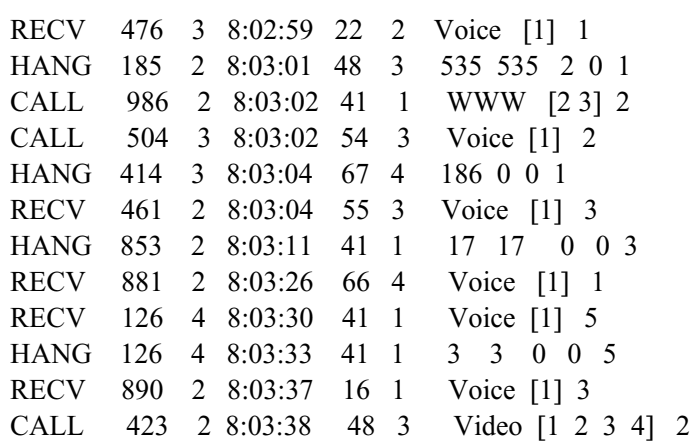

Figure 2. Simulation log file of acess to the $3 \mathrm{G}$ services.

mated from the variance of a statistical process. Selfsimilarity is implied if $0.5<H<1$. We used one graphical method to test the self-similarity of the traffic: variance-time plot $[1,21]$.

\begin{tabular}{|c|l|}
\hline Event & Description \\
\hline MOVE & $\begin{array}{l}\text { The MU must physically move within the city } \\
\text { area. }\end{array}$ \\
\hline RECV & The MU receives a call. \\
\hline CALL & The MU originates a call. \\
\hline HANG & $\begin{array}{l}\text { The call received or originated by an MU termi- } \\
\text { nates. }\end{array}$ \\
\hline HOFF & $\begin{array}{l}\text { The system must process handoff for a specific } \\
\text { MU from one cell to another. }\end{array}$ \\
\hline
\end{tabular}

Table 1. Events table

For the variance-time plot, the process is selfsimilar if the estimated asymptotic slope $\hat{\beta}$ is between -1 and 0 . The Hurst parameter can then be estimated as $\hat{H}=1-(\hat{\beta} / 2)$. We estimated the $\mathrm{H}$ value for one cell sample. The asymptotic slope estimated is aproximately -0.25 , resulting in an estimate $\hat{H} \approx 0.88$ of the Hurst parameter. This method suggests that the traffic sequence is self-similar.

The self-similar process has been constructed by superimposing several processes on/off ${ }^{2}$, where times between arrivals has a heavy tailed distribution It has influenced the blocking rate. So the process of Poisson used to model the arrival of voice service requests started by users, was not the same to the arrival of

\footnotetext{
${ }^{2}$ The periods on correspond to the periods of transmission of web files and the periods off correspond to the periods where the server is not receiving data
}

web documents in a server. Since the transmissions of WWW and video documents are not completely started by user. For instance, a web page often contains several images and when user demands one of these pages the browser automaticaly generates a lot of additional requests to read these images. Therefore the blocking rates of WWW and video services were bigger than voice service due to the used model, which allows to simulate another additional request needed to provide these type of services. Power-tails distributions have been used to model network traffic behavior [12].

\section{Data Traffic Modeling}

Intuitively, we consider heavy-tailed distributions as models for possibly large values in a sample. Such models have been taken as basis for more sophisticated models in teletraffic data transmission [11,28]. In certain applications, in particular in queueing theory, more structure for the distribution tail is needed. The Pareto distribution is characterized by a linearly increasing mean excess function. In addition, it has finite mean, infinite variance, and it has been used to model traffic having fractal characteristics [26].

The data traffic from the wireless network is modeled by Pareto distribution, where the traffic is generated by use of distribution inverse function. Like WAP (Wireless Application Protocol) data service has quickly developed [19], we have obtained from a wireless carrier of this service the average data traffic in the network. In this case, the average data traffic has been used in the simulation is $1.52 \mathrm{kbps}$.

Heavy-tailed service times, has been researched in $[3,11]$. Specifically, in [3] has been proved that when the service time is lighter than the tail of a Weibull distribution with parameter $\beta=0.5$, the number of arriving customers comes into the picture as well. Then the combination of the number of customers and the likely large service time the queuelength large.

The resource occupation time for data service is modeled by another heavy tailed distribution, called Weibull distribution. In the case of our simulation, we used the method of sucessive projections $[6,9]$ to estimate the shape parameters. Thus the Weibull distribution inverse function has been used to generate the 
occupation time with average of 2.52 minute $(\approx 180$ seconds).

The following subsection describes how the shape parameters were calculated for both Pareto and Weibull distributions.

\subsection{Calculating Shape Parmeter of Pareto Distri- bution}

There are in the literature a lot of methods that allow to estimate the $\alpha$ parameter value. Specifically, we used the Method of Moments, because this method does not need of an initial sample set. The sample average is defined as follows:

$$
M(x)=\frac{\alpha}{\alpha-1}
$$

Therefore, we can calculate $\alpha$.

$$
\begin{gathered}
\alpha=\frac{M(x)}{M(x)-1} \text { where } \\
M(x)=\text { average data traffic. }
\end{gathered}
$$

\subsection{Calculating Shape Parmeter of Weibull Distri- bution}

The method of sucessive projection has been used to determine parameters $\alpha$ and $\beta$ of the Weibull distribution. This method can be considered as a generalization, described in [2] [24][6] [9]. The $\alpha$ and $\beta$ values are obtained by the plans intersection in $\Re^{3}$, such that these values are greater than zero. The equations 3 and 4 represent the mean and the variance of Weibull distribution, respectively .

$$
\begin{gathered}
M=\alpha^{-\frac{1}{\beta}} \Gamma\left(1+\frac{1}{\beta}\right) \\
\sigma^{2}=\alpha^{-\frac{2}{\beta}}\left[\Gamma\left(1+\frac{2}{\beta}\right)-\Gamma^{2}\left(1+\frac{1}{\beta}\right)\right]
\end{gathered}
$$

This allows us to create a system as showed in Equation 5. Where $M \approx \bar{x}$ is the mean and the $\delta^{2} \approx S^{2}$ is the variance.

$$
\left\{\begin{array}{c}
\alpha^{-\frac{1}{\beta}} \Gamma\left(1+\frac{1}{\beta}\right)-\bar{x}=0 \\
\alpha^{-\frac{2}{\beta}}\left[\Gamma\left(1+\frac{2}{\beta}\right)-\Gamma^{2}\left(1+\frac{1}{\beta}\right)\right]-S^{2}=0
\end{array}\right.
$$

The Equations 6 and 7 can be determined applying logarithm properties.

$$
\beta=\frac{\log (\alpha)}{\log \left(\Gamma\left(1+\frac{1}{\beta}\right)\right)-\log (\bar{x})}
$$

$$
\alpha=2^{-\frac{\beta}{2}\left[\log \left(S^{2}\right)-\log \left[\Gamma\left(1+\frac{2}{\beta}\right)-\Gamma^{2}\left(1+\frac{1}{\beta}\right)\right]\right]}
$$

In this case, we know the mean $\bar{x}$, that is, the average occupation time for data service. By expanding 6 , we have 8):

$$
\beta \log \left(\Gamma\left(1+\frac{1}{\beta}\right)\right)-\beta \log (\bar{x})-\log (\alpha)=0
$$

The Equation 8 can be divided in two function, where $g(\alpha, \beta)$ is convex and $h(\alpha, \beta)$ is concave. They can be computed by 9 and 10 .

$$
\begin{gathered}
g(\alpha, \beta)=\beta \log \left(\Gamma\left(1+\frac{1}{\beta}\right)\right)+0 \alpha \\
h(\alpha, \beta)=\log (\alpha)+\beta \log (\bar{x})
\end{gathered}
$$

Therefore, we can write $f(\alpha, \beta)$ as shown in Equation 11.

$$
f(\alpha, \beta)=g(\alpha, \beta)-h(\alpha, \beta)
$$

The following hypothesis are necessary to determine $\bar{\alpha}$ e $\bar{\beta}$.

- $g(\alpha, \beta)$ convex

- $h(\alpha, \beta)$ concave

- $\exists \bar{\alpha}, \bar{\beta}: g(\bar{\alpha}, \bar{\beta})=h(\bar{\alpha}, \bar{\beta})$

Thus a linear approximation of $g(\alpha, \beta)$ in $(\bar{\alpha}, \bar{\beta})$ can be made. We refer $x=\left[\begin{array}{l}\alpha \\ \beta\end{array}\right]$. Then, we have a linear approximation $g(x)$ in $x_{k}$. Applying sucessive projection and making the intersection of two hyperplans we can determine $\bar{\alpha} \mathrm{e} \bar{\beta}$. Finally, the data service average occupation time can be generated.

\section{Simulation Results}

The simulation tool model both the traffic and mobility behaviour of users offering ability to consider different service types and different MU categories. Morever, it allows to estimate the blocking propability and the radio resource utilization. In particular:

- Voice and dada service are available anywhere.

- New calls and handoffs are differently treated.

- We consider 162 channel per cell. 
- The Simulation time is $24 \mathrm{~h}$.

- The Timeout to handoff queue is 2 seconds.

- The increase of the signal power occurs to 50 meters of cell's boundary.

- The Mean call from the originating and receiving rate for categories 1,2 and 3 is 0.9 calls/hour/MU.

- The Mean call originating and receiving rate for category 4 is 1.7 calls/hours/MU

- See Table 2 for the distribution of MUs among categories. The mobility parameter in the third column gives the time percentage that MU moves into the simulator period.

- The Mean duration for voice service is $120 \mathrm{sec}-$ onds, for data service is 180 seconds, and for video service is 3600 seconds.

- The MU speed varies between 30 and $50 \mathrm{~km} / \mathrm{h}$

We use 162 channels per cell, a parameter used by the American D-AMPS (Digital Advanced Mobile Phone System) or TDMA IS-54 ${ }^{3}$, which was standardized by FCC (Federal Communications Commission). The bands of $25 \mathrm{MHz}$ are divided into channels of 30 $\mathrm{KHz}$, that give us 416 duplex channels, with 21 for control. We apply a reuse factor of 7, for the 395 remainder, then we have 56 channels per cell. There are 3 time slots per carrier, that gives a service capacity of 168 channels or user per cell. Depending on the interference between channels, for example, this number can be decreased $(54 \times 3=162)$. In the case of GSM (Global System for Mobile Communications) we could consider until 192 channels per cell ( $3 \mathrm{sec}-$ tors, 8 carriers per sector, 8 slots per carrier).

\begin{tabular}{|c|c|c|c|}
\hline Category & \% of UMs & Mobility (\%) & Description \\
\hline 1 & 18.2 & 90 & $24 \mathrm{~h}$ delivery boy \\
\hline 2 & 35.3 & 50 & Common worker \\
\hline 3 & 26.3 & 30 & Housekeeper \\
\hline 4 & 20.2 & 60 & Taxis \\
\hline
\end{tabular}

Table 2. Categories and the MUs distribution

${ }^{3}$ Time Division Multiple Access was first specified as a standard in EIA/TIA (Electronic Industries Association) Interim Standard 54 (IS-54).

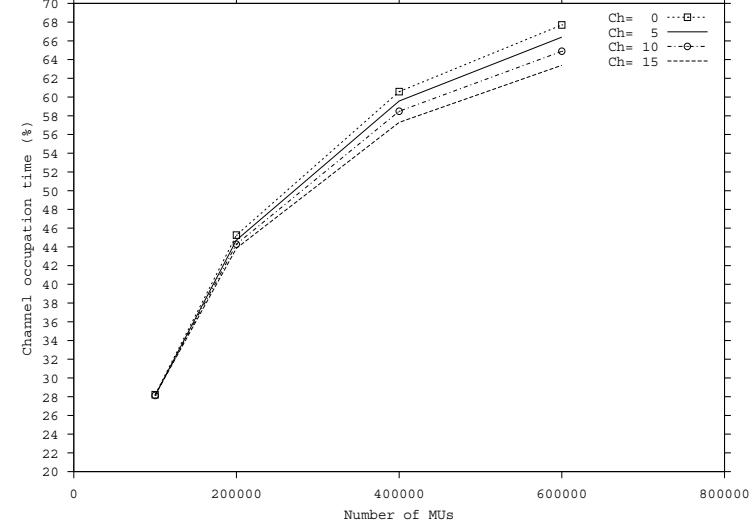

Figure 3. Channel occupation time in $3 G$ telecommunication networks per number of MUs per cell $(30 / 50 \mathrm{~km} / \mathrm{h})$

Figure 3 shows the influence of the number of MU in the effective channel occupation time with different variations of reserved channels to the handoff procedure. We see that when the number of MUs is increased, this will mean that more calls being held by each cell, leading to a better channel occupancy. Relatively, we can also note that a variation on number of reserved channels to handoff does not interfere in the channel occupation time.

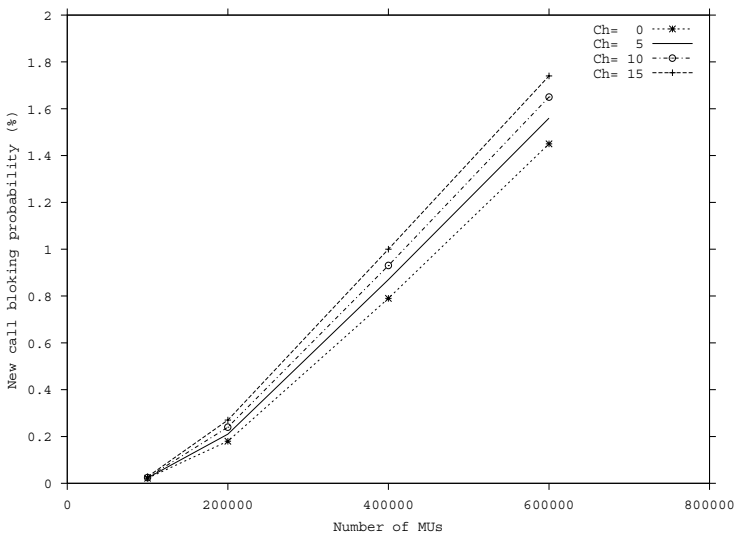

Figure 4. New call blocking probability in 3G telecommunication networks per number of MUs per cell $(30 / 50 \mathrm{Km} / \mathrm{h})$

One of the main parameters used to measure QoS (Quality of service) is the blocking probability, i.e., the number of calls rejected because of lack in channel ca- 
pacity. In practice, this value should not exceed $2 \%$ for new calls. In Figure 4 we observe that when the number of channels to handoff is increased, the new call blocking probability also raises. If we compare the new call blocking probability between $3 \mathrm{G}$ and current telecomunication networks, we could note a high blocking probability in $3 \mathrm{G}$ network. This happens because the same channels of current networks are used in $3 \mathrm{G}$ networks to move beyond of voice service, the video and data services. This implies in a larger consumption of resources.

Specifically, there is a reduction in the number of channels available to answer new calls in the system in such way to attend the requests of handoff. If we consider that the number of MU has a tendency to remain either constant or increase, the new call blocking will increase. On the other hand the MUs can perform more handoff, but also the QoS of the network is damaged.

Figure 5 shows a new call blocking probability per service (voice, video, WWW), changing the number of reserved channels to handoff. We can note that the WWW and video services blocking rates are greater than voice service blocking rate when the number of reserved channels to handoff increases. This result may be explained by the fact that video connection requires a larger amount of resources to maintain the minimum QoS and the connection percentage is small. On the other hand, for the WWW service, the amount of the resource necessary is smaller than the video resources, but the connection percentage is bigger.

Besides that, the 162 available channels to meet the demand of new calls are decreased in the same amount of with the reserved channels to handoff. In addition, the low blocking probability of voice service may be explained by the fact that this service uses only one channel for each connection.

The simulator mobility model tries to control a blocking probability per cell around $1 \%$. Considering different number of reserved channels to handoff call, Figure 6 indicates that as the number of MUs increases and the number of reserved channels to handoff decreases, the handoff blocking probability also increases. We can also note that the handoff blocking probability in $3 \mathrm{G}$ network tends to 0 when the number of reserved channels to handoff procedure are greater or equal 5. In this case, the superimposition of curves

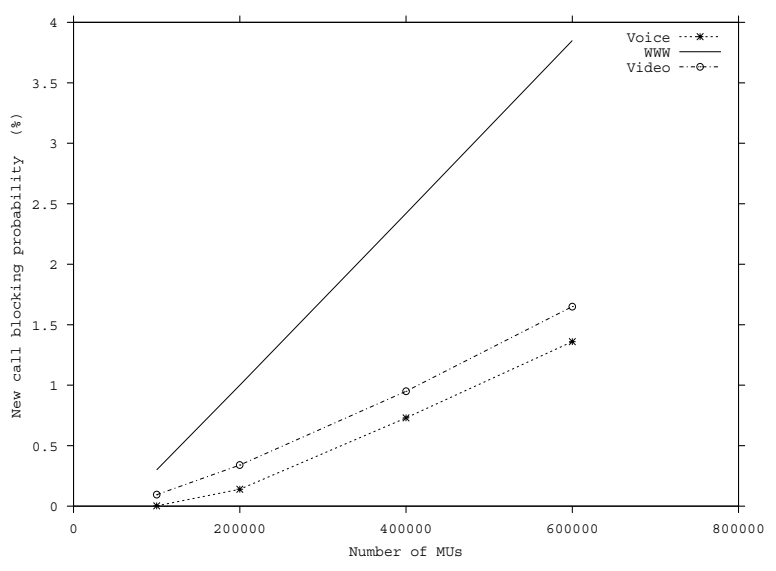

(a) Ch. Handoff $=0$

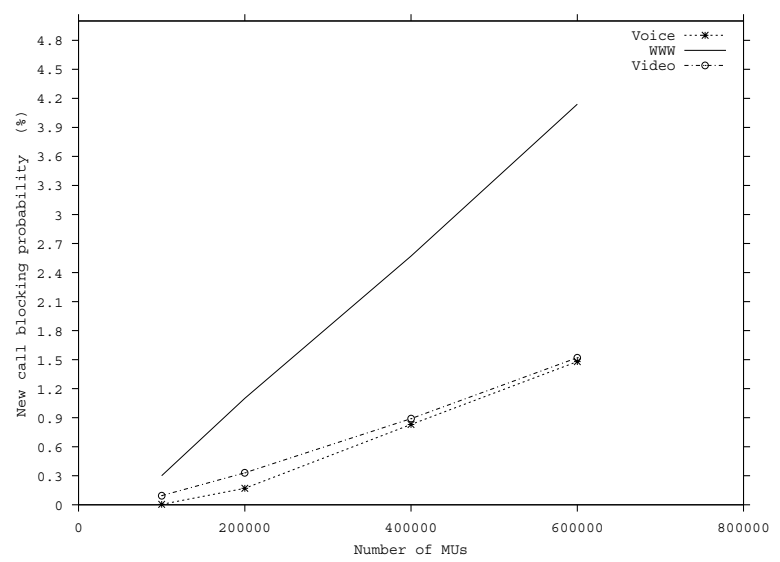

(b) Ch. Handoff $=5$

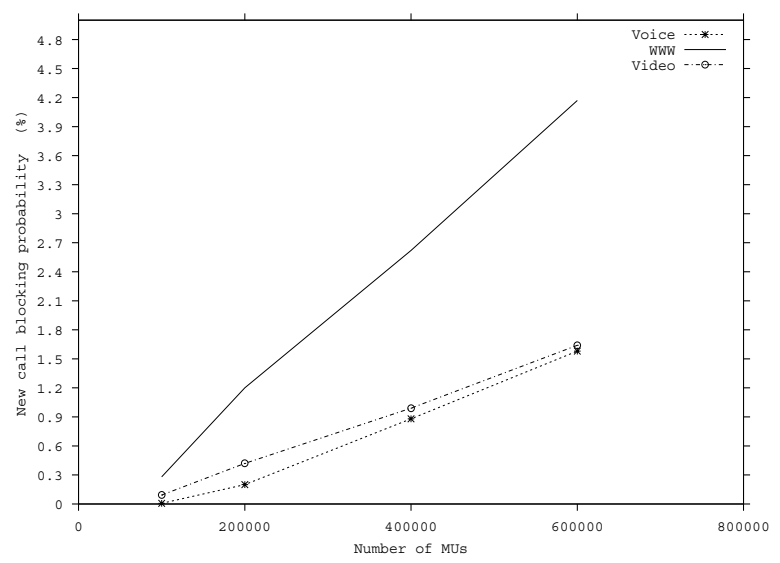

(c) Ch. Handoff $=10$

Figure 5. New call blocking probability in 3G telecommunication networks per type of service per cell( $30 / 50 \mathrm{Km} / \mathrm{h})$ 


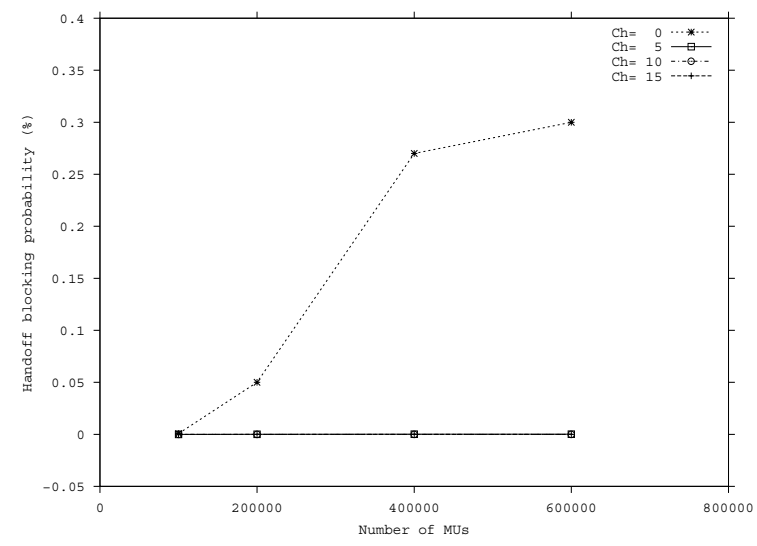

Figure 6. Handoff blocking probability in 3 G telecommunication networks per number of MUs per cell $(30 / 50 \mathrm{Km} / \mathrm{h})$

occurs as depicted in Figure 6.

In Figure 7, we analyze the variation of MUs speed fixing the number of channels to handoff equal 5 . We can note how the MU mobility degree, might influence in the quality of service of the network. We observe that as the MU's speed and the number of MUs increases towards high values, the handoff blocking probability raises.

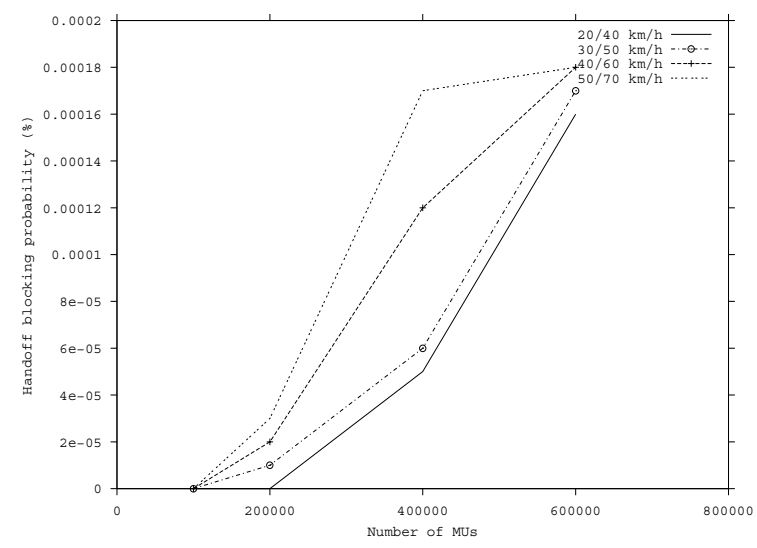

Figure 7. Handoff blocking probability in $3 G$ telecommunication networks per number of MUs per cell changing MU's speed

Figure 8 shows the handoff blocking probability in $3 \mathrm{G}$ network per service with MU speed between 30 and $50 \mathrm{Km} / \mathrm{h}$, changing the reserved channels to handoff. When the number of reserved channels to handoff is increased, the blocking probability reduces. In this case, the number of MUs must remain unchanged.

We also see that both voice and WWW service blocking probability tends to 0 for handoff channels greater or equal 10 . While the video service blocking probability tends to $2.0 * 10^{-5}$, so it require larger amount of resources, in agreement with established criteria in the simulation.

We also note that rising the number of handoff channels, the carried traffic tends to decrease. This result can be explained by the fact that the number of channels to serve new call is decreased, while we increase the number of reserved channels to handoff.

If we vary the MUs speed and fix the number of available handoff channels in 5 , then we observe that, independent of MU speed, the carried traffic (in Erlangs) keep unchanged.

Specifically, when we increase the number of MU at system the carried traffic varies clearly. On the other hand, a increasing in the number of available channels to handoff procedure, causes a reduction in the number of available channels to new call, but also there is a reduction of the performed handoff number. In this case the traffic volume remains stable, but also there is no guarantee of quality to users demanding access to services.

Figure 9 shows the carried traffic (in Erlangs) per cell. In this case, we vary the number of reserved channels to handoff procedure. We can note that rising the number of handoff channels, the carried traffic tends to decrease. This result can be explained by the fact that the number of channels to serve new calls are reduced, while we increase the number of reserved channels to handoff.

If we vary the MUs speed as is showed in Figure 5 and fix the number of available handoff channels in 5, then we observe that, independent of MU speed, the carried traffic (in Erlangs) keep unchanged as we can note by the superimposition of the curves.

Specifically, when we increase the number of MU's at system the carried traffic varies clearly. On the other hand, a increase in the number of available channels to handoff procedure, causes a reduction in the number of available channels to new calls, but also there is a reduction of the performed handoff number. In this 


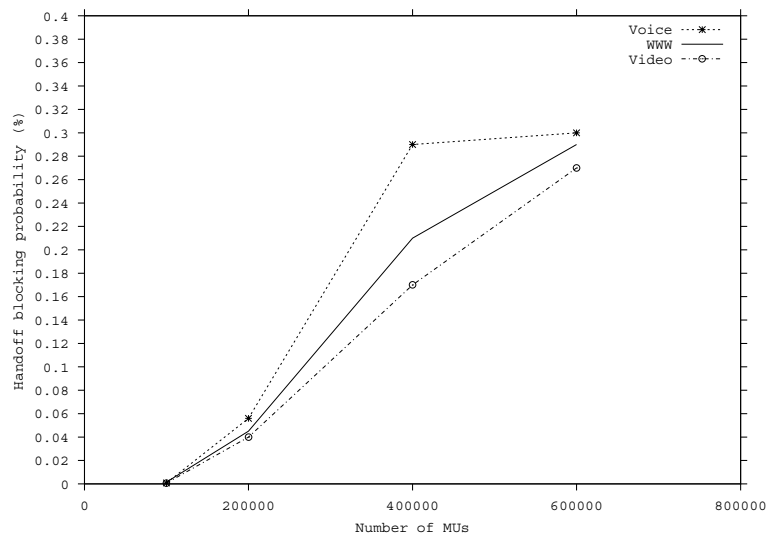

(a) $\mathrm{Ch}$. Handoff $=0$

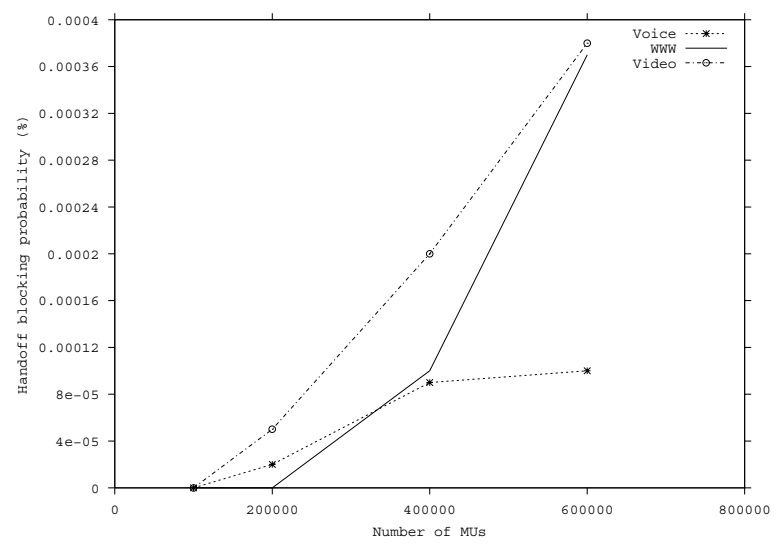

(b) Ch. Handoff $=5$

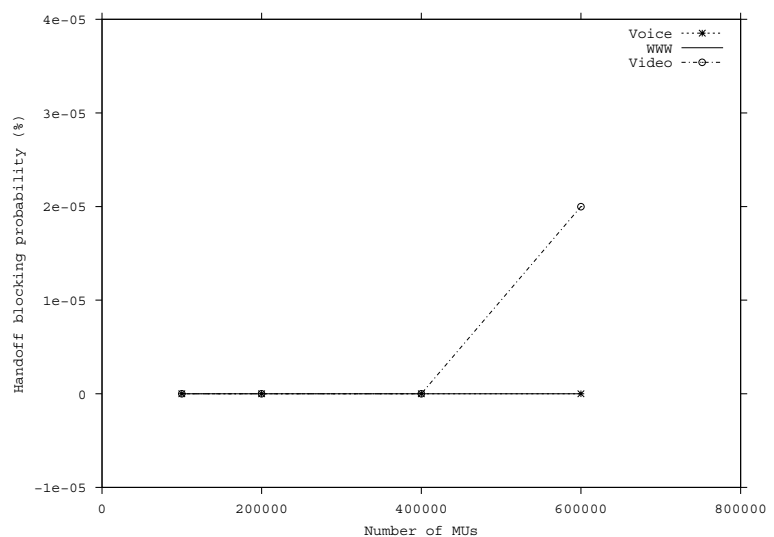

(c) Ch. Handoff $=10$

Figure 8. Handoff blocking probability to $3 G$ mobile telecommunication network per service per cell $(30 / 50 \mathrm{Km} / \mathrm{h})$

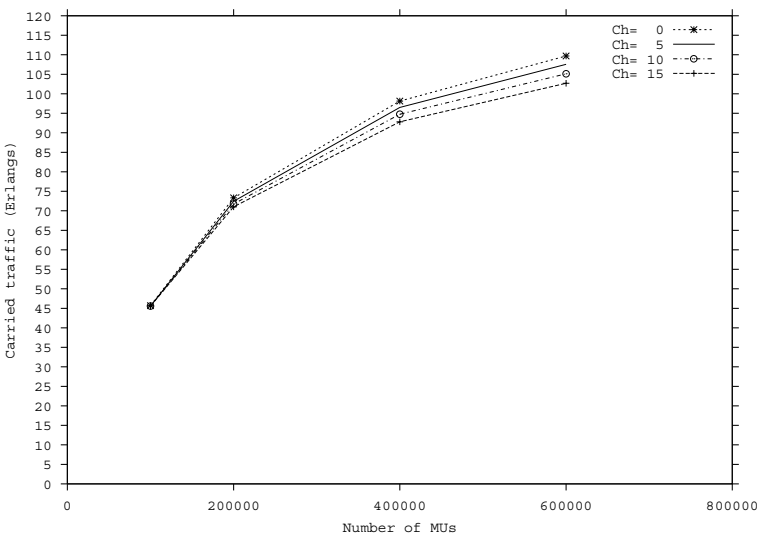

Figure 9. Carried traffic (in Erlangs) per cell to mobile telecommunication networks (30/50 $\mathrm{Km} / \mathrm{h}$ )

case the traffic volume remains stable, but also there is no guarantee of quality to users who are demanding access to the services.

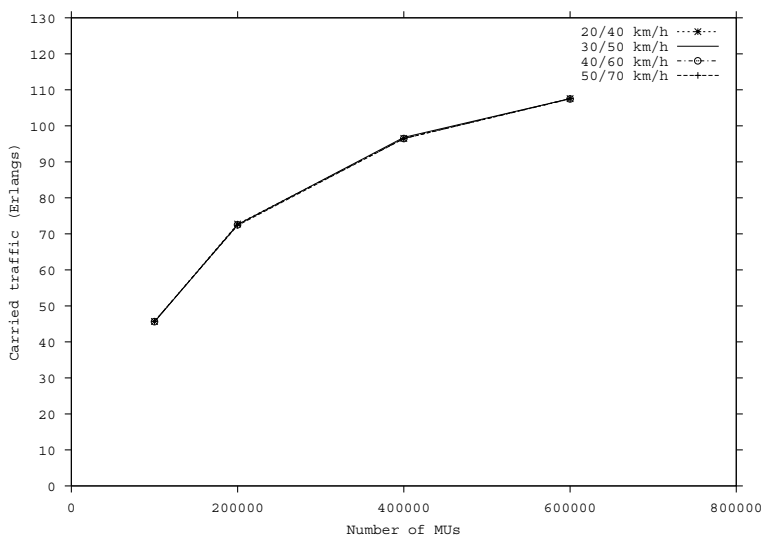

Figure 10. Carried traffic (in Erlangs) per cell to mobile telecommunication networks for different MUs speeds

Figure 11 shows the carried traffic (in Erlangs) to $3 \mathrm{G}$ networks per category of MU with inner/outer speed $30 / 50 \mathrm{Km} / \mathrm{h}$ with variation of the reserved channels to handoff. We can note that the carried traffic per category is little influenced by changes in the available channels to handoff. 


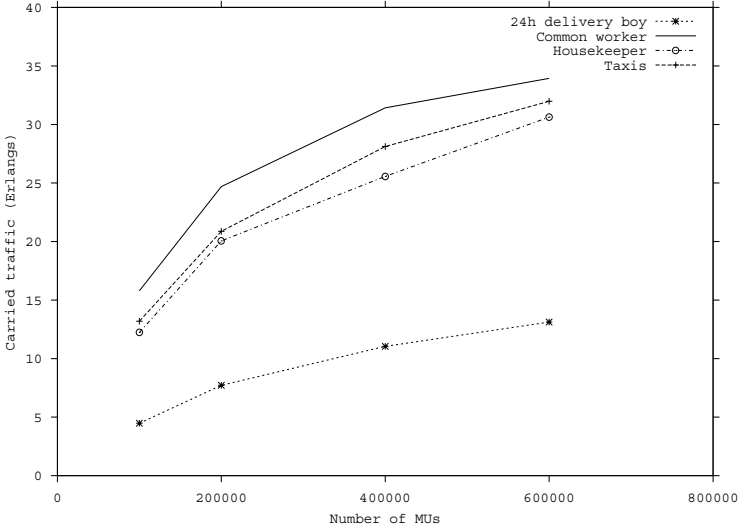

(a) Ch. Handoff $=0$

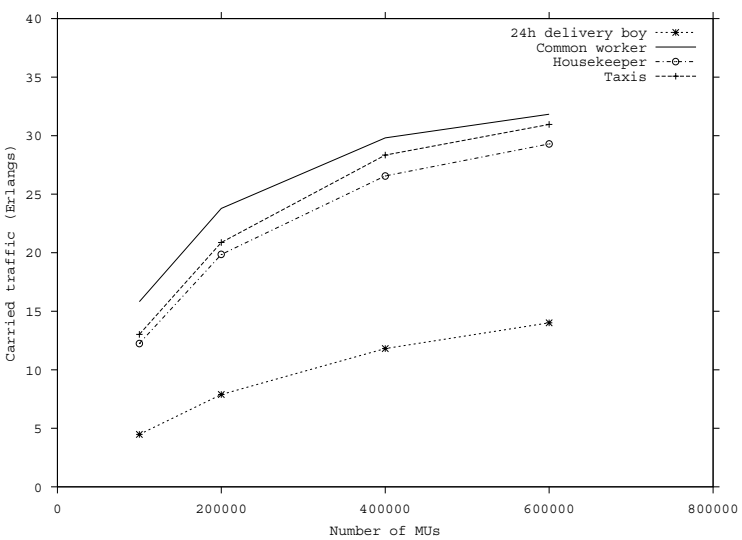

(b) Ch. Handoff $=5$

Figure 11. Carried traffic (in Erlangs) per category per cell to mobile telecommunication networks

We can still verify that the category 2 (common worker) tends to have a larger traffic, that is delineated by the large number of connection performed to the $3 \mathrm{G}$ services for this type of user. This implicates in a rising on channel occupation time, generating a rise in the traffic volume of network.

We observe that carried traffic in the network does not depend on available channels to handoff. We still note that the tendency is that the voice service traffic is bigger than the video and WWW services traffic. This happens because a large number of requests is generated by voice services. These requests can use any of the 162 channels for a generated new call, they can decrease when we increase the number of reserved channels to handoff. In this case, the new call blocking probability increases, reducing the traffic volume in the network. This traffic volume depend on channel occupation time, what imply in a reduction of the carried traffic.

\section{Conclusions}

In this paper we have presented a performance analysis of third generation mobile telecommunication networks, using a simulator. We have considered some parameters, such as new call and handoff blocking probability, carried traffic (in Erlangs), and channel occupation time that make possible evaluate the QoS of mobile networks. Traffic analysis for future mobile systems should take into account a variety of services (voice, data, video) and environments (e.g.: private, outdoors, indoors) as well as the user mobility behavior. The simulator presented in this paper incorporates all the above mentioned features, besides it considers the self-similar network traffic (in this case we have used the heavy tailed distribution to simulate self-similarity of data traffic). In the case of the voice service: the call duration has been exponentially distributed and the arrival process of new calls have been Poissonian.

These results clearly show that the mobility directly influences in the network quality of service and that the different available types of services depend on other issues as carried traffic, bandwidth, etc. These features are essential part of mobile communication system design. Each type of traffic has different characteristics. Then, the capacity planning becomes a hard task with a variety of data applications, such as Internet access, email, file transfer, and with a variety of data rates and mix of traffic. Hence, in $3 \mathrm{G}$ network planning is crucial to use capacity planning techniques which give accurate results, because it directly affects the number of cells required to serve a given area. The simulation is one of the aspects that make possible the wireless provider to prepare for $3 \mathrm{G}$ wireless networks.

Future research must focus on methods to reduce the blocking probability and to maximize the utilization of the bandwidth according to the traffic and type of service. Priority techniques must be included in 
the simulator to consider QoS constraints and channels dynamic allocation tecniques.

\section{References}

[1] G. Abdula and et al. Shared user behaivor on the word wide web. http://www.cs.vt.edu/ chitra/docs/97webnet, 1997.

[2] S. Agmon. The relaxation method for linear inequalities. In Can. J. Math, volume 6, pages 382-392. 1954.

[3] S. Asmussen, C. Kluppelberg, and K. Sigman. Sampling at subexponential times, with queueing applications, 1999. Stoch. Proc. Appl.

[4] A. Bar-Noy and L. Kessler. Tracking mobile users in wireless networks. In IEEE Trans. On Information Theory, volume 39, pages 1877-1886, November 1993.

[5] J. Beran, R. Sherman, M. S. Taqqu, and W. Willinger. Long-range dependence in variable-bit-rate traffic. IEEE Transactions on Communications, 1995.

[6] L. M. Breg. Finding the common point of convex sets by the method of sucessive projections. In Dokl. Akad. Mousk SSSR, pages 487-490. 1965.

[7] M. E. Crovella and A. Bestavros. Self-similarity in world wide web traffic: Evidence and possible causes. In Proceedings of SIGMETRICS Conference on Measurement and Modeling of Computer Systems, 1996.

[8] D. E. Duffy, A. A. McIntosh, M. Rosenstein, and W. Willinger. Statistical analysis of CCSN/SS7 traffic data from working subnetworks. IEEE J. Select Areas Commun, 1(3):544-551, 1994.

[9] I. I. Eremin. Generalization of the Motzkin - Agmon relaxational method. In Usp Math. Monk 20, pages 182-187. 1965.

[10] A. Erramilli, O. Narayan, and W. Willinger. Experimental queueing, analysis with long-range dependent pocket traffic. IEEE/ACM Trans. Networking, 4(2), 1996.

[11] M. Greiner, M. Jobmann, and C. Kluppelberg. Telecommunication traffic, queueing models, and subexponential distributions. Advances in Computacional Mathematics, 1999. Departament of Computer Science, Munich University of Technology.

[12] M. Greiner, M. Jobmann, and L. Lipsky. The importance of power-tail distributions for telecommunications traffic models. Technical report, Institute für Informatik, Technische Universität Munchen, 1995.

[13] R. Guri. Channel occupancy time distribution in a cellular radio system. In IEEE transactions on Vehicular Technology, volume 36, pages 89-99. August 1987.

[14] D. Hong and S. S. Rappaport. Traffic model and performance analysis for cellular mobile radio telephone systems with prioritized and nonprioritized handoff procedure. In IEEE transactions on Vehicular Technology, volume 35, pages 77-92. August 1986.
[15] D. Hong and S. S. Rappaport. Traffic model and performance analysis for celular mobile radio telephone systems with prioritized and nonprioritizied handoff procedures. In IEEE Transaction on Vehicular Technology, volume 35, pages 77-92, August 1986.

[16] B. Jabbari. Teletraffic aspects of evolving and next generation wireless communication networks. In IEEE Transactions on Vehicular Technology, volume 3, pages 4-9. December 1996.

[17] R. Jain. The Art of Computer Systems Performance analysis, techniques for Experimental Design, Measurement, Simulation, and Modeling. John Wiley \& Sons, INC, 1991.

[18] M. Jiang, M. Nikolic, S. Hardy, and L. Trajkovic. Impact of self-similarity on wireless data network performance. In IEEE International Conference on Communications, June 2001.

[19] T. Kunz, T. Barry, J. P. Black, and H. M. Mahoney. WAP traffic: Description and comparison to WWW traffic. In 3rd ACM International Workshop on Modeling Analysis and Simulation of Wireless and Mobile Systems, August 2000.

[20] W. E. Leland, M. S. Taqqu, W. Willinger, and D. V. Wilson. On the self-similar nature of ethernet traffic. ACM Sigcomm Computer Communications Review, 23:183-193, 1993.

[21] W. E. Leland, M. S. Taqqu, W. Willinger, and D. V. Wilson. On the self-similar nature of ethernet (extended version). IEEE/ACM transactions on Networking, 2(1):1-15, February 1994.

[22] J. G. Markoulidakis, G. L. Lyberopoulos, and M. E. Anagnostou. Traffic model for third generation cellular mobile telecommunication systems. In Wireless Networks, pages 389-400. 1998.

[23] M. Middendorf. Manhattan channel routing is NPcomplete under truly restricted settings. Chigago Journal of Theorical Computer Science, 2:1-19, 1996.

[24] T. S. Motskin and I. I. Schoenberg. The relation method for linear inequalities. In Can. J. Math, volume 6, pages 393-404. 1954.

[25] R. Pandya, D. Grillo, and P. Mieybegue. IMT-2000 standards: Network aspects. In IEEE Personal Communications, pages 20-29. August 1997.

[26] P. Ramakrishnan. Self-similar traffic model. Technical Report CSHCN T.R.99-5 (ISR T.R. 99-12), Center for Satellite and Hybrid Communication Networks, 1997. www.isr.umd.edu/CSHCN/.

[27] J. Rapeli. UMTS: Targets, system concept, and standardisation in a global framework. In IEEE communications, pages 20-28, February 1995.

[28] S. I. Resnick. Heavy tail modeling in teletraffic data. In Ann. Statist., number 25, pages 1805-1848. 1997. 
[28] S. I. Resnick. Heavy tail modeling in teletraffic data. In Ann. Statist., number 25, pages 1805-1848. 1997.

[29] M. N. Rocha, G. R. Mateus, and S. L. Silva. A comparison between location updates and location area paging for mobile unit tracking simulation in wireless communication systems. In The 3rd Int. Workshop on Discrete Algorithms and Methods for Mobile Computing and Communications (DialM-99), pages 72-77, Seattle, WA, August 1999.

[30] M. N. Rocha, G. R. Mateus, and S. L. Silva. Simulation of mobile unit tracking simulation in wireless communication systems. In Wireless Personal Multimedia Communications (WPMC'99) Conference, Amsterdam, The Netherlands, September 1999.

[31] M. N. Rocha, G. R. Mateus, and S. L. Silva. Traffic simulation and the location of mobile units in wireless communication systems. In 17th Brazilian Computer Networks Symposium, pages 405-417, May 1999.

[32] A. Samnkic. UMTS universal mobile telecommunications system: Development of standard for the third generation communications. In IEEE Transactions on Veicular Technology, volume 47, pages 1099-1104. November 1998.

[33] M. Sidi and D. Starobinski. New call blocking versus handoff blocking in cellular networks. In Wireless Networks, volume 3, page 1 27. February 1997.

[34] A. P. Silva and G. R. Mateus. Performance analysis for data service in third generation mobile telecommunication networks. In Advanced Simulation Technologies Conference 2002 - 36th Annual Simulation Symposium, pages 227-235. IEEE and Computer Society, April 2002.

[35] X. Zhou. Cellular data traffic: Analysis, models, and scenarios. Master's thesis, Ottawa-Carleton Institute for Computer Science, Carleton University, June 2000. 